\title{
Vascular events, mortality, and preventive therapy following ischemic stroke in the elderly
}

\author{
R.C. Kaplan, PhD; D.L. Tirschwell, MD; W.T. Longstreth, Jr., MD, MPH; T.A. Manolio, MD, PhD; \\ S.R. Heckbert, MD, PhD; D. Lefkowitz, MD; A. El-Saed, MD; and B.M. Psaty, MD, PhD
}

\begin{abstract}
Background: The authors studied mortality, vascular events, and preventive therapies following ischemic stroke among adults aged $\geq 65$ years. Methods: The authors identified 546 subjects with first ischemic stroke during 1989 to 2001 among Cardiovascular Health Study participants. Deaths, recurrent strokes, and coronary heart disease (CHD) events were identified over 3.2 years (median) follow-up. Results: During the first year of follow-up, rates were 105.4/1,000 for recurrent stroke and 59.3/1,000 for CHD. After the first year, the stroke rate was 52.0/1,000 and the CHD rate was 46.5/1,000. Cardioembolic strokes had the highest mortality (185.4/1,000) and recurrence rates (86.6/1,000). Lacunar strokes had the lowest mortality (119.3/1,000) and recurrence rates (43.0/1,000). Age and male sex predicted death and CHD, but not recurrence. Outcomes did not differ by race. Following stroke, 47.8\% used aspirin and $13.5 \%$ used other antiplatelet agents; $52.6 \%$ of patients with atrial fibrillation used warfarin; $31.3 \%$ of hyperlipidemic subjects, $57.0 \%$ of diabetic patients, and $81.5 \%$ of hypertensive patients were drug-treated; and $40.0 \%$ of hypertensive patients had blood pressure (BP) $<140 / 90 \mathrm{~mm} \mathrm{Hg}$. Older subjects were less likely to use lipid-lowering therapy, women were less likely to have $\mathrm{BP}<140 / 90 \mathrm{~mm} \mathrm{Hg}$, and low-income subjects were less likely to use diabetes medications. Conclusions: Recurrent strokes were nearly twice as frequent as coronary heart disease (CHD) events during the first year after initial stroke, but stroke and CHD rates were similar after the first year. Preventive drug therapies were underused, which may reflect clinical uncertainty due to the lack of clinical trials among the elderly. Utilization was lower among the oldest patients, women, and low-income individuals.
\end{abstract}

NEUROLOGY 2005;65:835-842

Ischemic stroke is a major source of morbidity and mortality among older adults. Between the 1970s and $1990 \mathrm{~s}$, the number of noninstitutionalized stroke survivors in the 60 - to 74-year-old US population increased from 917,000 to $1,475,000 .{ }^{1}$ At present, more than $5 \%$ of individuals 65 to 74 years old and more than $10 \%$ of those 75 years and older have had a prior stroke. ${ }^{2}$ Rates of death and cardiovascular disease (CVD) events are high after ischemic stroke. However, prognosis among elderly populations of stroke survivors is not well-described. In particular, while several studies have examined mortality and recurrent stroke, ${ }^{3-7}$ there are fewer studies of coronary events after stroke. ${ }^{8-10}$ Preventive therapies such as antihypertensive medications, lipid-lowering

Additional material related to this article can be found on the Neurology Web site. Go to www.neurology.org and scroll down the Table of Contents for the September 27 issue to find the title link for this article. therapy, aspirin, and oral anticoagulants are underutilized among stroke survivors. ${ }^{11-14}$ Few recent datasets from the United States describe use of therapies and factors associated with underutilization among older adults with ischemic stroke.

We conducted a study of long-term prognosis and use of preventive medical therapies following first ischemic stroke among participants in the Cardiovascular Health Study (CHS) cohort of older adults. We assessed the subsequent occurrence of death and CVD events, examined the use of CVD preventive therapies, and identified risk factors for morbidity, mortality, and underutilization of medical therapies after ischemic stroke.

Methods. Study population and setting. CHS is a prospective population-based cohort study of stroke and other CVD in adults 65 years and older living in four US communities. ${ }^{15}$ The original cohort of 5,201 participants was recruited in 1989 to 1990. In 1992 to 1993 , 687 additional participants were recruited, almost all of

From the Department of Epidemiology and Population Health (Dr. Kaplan), Albert Einstein College of Medicine, Bronx, NY; Departments of Neurology (Drs. Tirschwell and Longstreth), Epidemiology (Drs. Longstreth, Heckbert, and Psaty), Medicine (Dr. Psaty), and Health Services (Dr. Psaty), University of Washington, Seattle; Epidemiology and Biometry Program (Dr. Manolio), National Heart, Lung, and Blood Institute, National Institutes of Health, Bethesda, MD; Department of Neurology (Dr. Lefkowitz), Wake Forest University School of Medicine, Winston-Salem, NC; and Department of Epidemiology (Dr. El-Saed), University of Pittsburgh.

Supported by contracts N01-HC-85079 through N01-HC-85086, N01-HC-35129, and N01 HC-15103 from the National Heart, Lung, and Blood Institute and grant 5R01AG009556-08 from the National Institute on Aging.

Disclosure: The authors report no conflicts of interest.

Received January 26, 2005. Accepted in final form June 6, 2005

Address correspondence and reprint requests to Dr. Robert C. Kaplan, Department of Epidemiology and Population Health, Belfer Building, Room 1303A, Albert Einstein College of Medicine, 1300 Morris Park Avenue, Bronx, NY 10461; e-mail: rkaplan@aecom.yu.edu 
whom were African American, in order to enhance the racial/ ethnic diversity of the cohort. Potential subjects were identified from Medicare eligibility lists of the Health Care Financing Administration (HCFA). ${ }^{15}$ Those eligible to participate included all persons living in the household of each individual sampled, who 1) were 65 or older at the time of examination; 2) expected to remain in the area for 3 years; and 3) were able to give informed consent. Among those contacted and eligible, $57.3 \%$ were enrolled.

Study visits, questionnaires, and laboratory assays. CHS participants completed standardized clinic examinations and questionnaires at study baseline and at 9 annual follow-up visits. ${ }^{15}$ Subjects who were unable to attend their local CHS clinic were offered the opportunity to complete study visits in their own home or nursing home. In addition, follow-up for mortality and CVD events was continued through phone contacts and national database searches for all subjects including those who did not continue study visits. Questionnaires included an assessment of medical history, health-related behaviors, and demographic and socioeconomic factors. Physical examinations included repeat right-arm blood pressures (BPs) and 12-lead resting EKGs. ${ }^{16}$ Current prescription medication use was assessed using an inventory method. ${ }^{17}$ Direct questions about the use of aspirin (frequency of use within prior 2 weeks) were also asked as part of the medication inventory. Fasting lipids and glucose were measured at the baseline, year 3 , and year 7 visits. ${ }^{18}$

Definition of CVD risk factors. Prior CHD was defined as history of myocardial infarction (MI) or angina pectoris (including coronary revascularization). Hypertension was defined as prior physician diagnosis of hypertension with or without use of antihypertensive drugs. Diabetes was defined as fasting glucose $>=126$ $\mathrm{mg} / \mathrm{dL}$ or physician diagnosis of diabetes. Hyperlipidemia was defined as LDL-cholesterol $>=4.1 \mathrm{mmol} / \mathrm{L}(160 \mathrm{mg} / \mathrm{dL})$ or use of lipid-lowering medications. Atrial fibrillation was defined based on study resting EKGs, which were read centrally. ${ }^{16}$

Collection of CVD events. All deaths and incident CVD events during follow-up were identified through semi-annual participant contacts, notification of events by participants, and periodic searches of national administrative databases (e.g., HCFA Medicare Utilization database, National Death Index). Medical records for all deaths and CVD events were centrally reviewed. ${ }^{19}$ Neuroimaging studies were available for $86 \%$ of suspected TIAs and strokes. Classification of major stroke types (i.e., ischemic or hemorrhagic) and etiologic subtypes of ischemic stroke was performed using criteria adopted from the SHEP study. ${ }^{20}$ Cardioembolic strokes were defined by autopsy evidence for embolus to the brain, or a potential source of cardiac emboli such as atrial fibrillation or flutter, MI within 6 weeks of the onset of stroke, an akinetic myocardial segment, intracardiac thrombus, valvular vegetation, prosthetic heart valve, dilated cardiomyopathy, right to left intracardiac shunt, or systemic embolus. Atherosclerotic large-artery strokes were defined according to the presence of a severe atherosclerotic stenosis $(>70 \%)$ or occlusion in a large vessel appropriate to the infarct as evidenced by noninvasive testing, angiography, or surgical or autopsy evidence. Lacunar strokes were defined by a lack of evidence for large vessel disease and a clinical presentation and imaging consistent with a lacunar syndrome. Ischemic strokes were classified as indeterminate/unknown etiology if they did not meet criteria for other subtypes or if multiple potential etiologies were identified. A reliability study found kappa $=0.86$ for stroke vs no stroke, kappa $=1.0$ for ischemic vs hemorrhagic stroke, and kappa $=0.77$ for definition of ischemic stroke subtypes, suggesting substantial reliability for classification of stroke. ${ }^{21}$

Inception cohort of ischemic stroke patients. For the analyses presented in this report, we identified an inception cohort of CHS participants who sustained a first hospitalized ischemic stroke between study baseline (1989/90 or 1992/93) and July 1, 2001. There were 705 CHS subjects with nonfatal stroke and 112 with fatal stroke during follow-up, not including nonhospitalized strokes and TIAs. Of the non-fatal strokes, we excluded 56 hemorrhagic strokes and 76 strokes that were indeterminate between ischemic and hemorrhagic stroke. Of the 573 ischemic strokes, we excluded 27 who had had a history of stroke at CHS study baseline. A total of 546 subjects with incident ischemic stroke were included in these analyses.

Identification of prestroke and poststroke visits. For each subject with incident ischemic stroke, risk factors and medications were assessed at both the CHS visit immediately prior to stroke ("prestroke visit") and at the next CHS visit completed after stroke ("poststroke visit"). While all stroke subjects were included in analyses of subsequent mortality and CVD events, only subjects who completed poststroke visits were included in analyses of medication use after stroke.

Analyses of mortality and CVD events. We examined the occurrence following ischemic stroke of 1) death, 2) recurrent stroke, and 3) CHD events. The outcome of death included all-cause mortality, including fatal stroke and CHD events. Recurrent stroke included fatal and non-fatal ischemic and hemorrhagic events. CHD events included fatal and non-fatal myocardial infarction and deaths attributed to CHD. We plotted survival curves and smoothed annualized hazard curves. Multivariate Cox proportional hazards regression was used to estimate adjusted hazard ratios and 95\% CIs for potential risk factors including stroke subtype, age, sex, race, prior CHD, systolic and diastolic BP levels, hypertension, diabetes, atrial fibrillation, total, LDL-, and HDLcholesterol levels, and current smoking. Total cholesterol, which was the most predictive lipid variable, and presence vs absence of hypertension, which was the most predictive BP variable, were selected for final models. For these analyses, all risk factors were defined at the prestroke visit, with the exception of atrial fibrillation, glucose levels, and cholesterol levels. In order to permit more sensitive assessment of atrial fibrillation, we defined subjects as having atrial fibrillation if either the prestroke visit EKG or the poststroke visit EKG indicated the presence of atrial fibrillation. Because glucose and cholesterol levels were not assessed at each study year, we used the most recent measurements available prior to stroke. Occasionally, values were missing from the visit immediately preceding stroke (BP level, $\mathrm{n}=55$ missing; smoking, $\mathrm{n}=$ 10 missing). In these instances, we carried forward data that were collected at earlier visits.

Analyses of preventive drug therapy use following stroke. Among subjects who completed a poststroke visit, we examined use of aspirin (daily use in prior 2 weeks), other antiplatelet agents (thienopyridines and dipyridamole), and warfarin after stroke. In addition, for subjects with the appropriate indications, we examined lipid-lowering medication use, diabetes medication use, antihypertensive medication use, and control of hypertension to below 140/90 $\mathrm{mm} \mathrm{Hg}$. Indications for preventive therapies were defined at the poststroke visit. We assessed potential predictors of medication use including year of stroke, study clinic, age, sex, race, history of $\mathrm{CHD}$, and household income greater or less than $\$ 12,000 / y e a r$. For analyses of antiplatelet therapy and warfarin use, we also examined atrial fibrillation and stroke subtype as potential predictors. Multivariate logistic regression models that included all predictors simultaneously were used to estimate adjusted ORs and 95\% CIs. Because of the high correlation between race/ethnicity and income, we alternated the inclusion of these two variables in multivariate models to avoid variance inflation. Subjects with values that were missing from the poststroke visit were excluded from these analyses (income, $\mathrm{n}=29$ missing; diabetes, $\mathrm{n}=11$ missing; $\mathrm{BP}$ level, $\mathrm{n}=49$ missing).

Results. Subject characteristics. Of 5,639 CHS participants without a baseline history of stroke, 546 sustained an incident hospitalized non-fatal ischemic stroke $(9.7 \%$ of subjects). There were 66 lacunar strokes, 104 cardioembolic strokes, 22 atherosclerotic large-artery strokes, and 354 indeterminate strokes (table 1). Mean age at the occurrence of stroke was 80.0 years (range 66 to 97 years), and $47 \%$ of subjects were over 80 years old. Of 71 subjects who reported race or ethnic group other than white, 69 were identified as African American. Other characteristics at the most recent visit prior to stroke appear in table 1 .

Mortality and vascular events. Over 3.2 years (median) of follow-up, $57.0 \%$ of study subjects $(\mathrm{n}=313)$ died, $22.0 \%(\mathrm{n}=120)$ sustained a recurrent stroke, and $17.4 \%$ ( $\mathrm{n}=95$ ) sustained a subsequent CHD event (figure 1A). The estimated hazard curves indicated a progressive decrease in stroke risk as time elapsed since the initial stroke, from an annualized risk of approximately $10 \%$ in 


\begin{tabular}{|c|c|c|c|c|c|c|c|}
\hline & \multicolumn{2}{|c|}{$\begin{array}{l}\text { All subjects, } \\
\mathrm{n}=546\end{array}$} & \multicolumn{2}{|c|}{$\begin{array}{l}\text { Without poststroke visits, } \\
\qquad \mathrm{n}=222\end{array}$} & \multicolumn{2}{|c|}{$\begin{array}{l}\text { With poststroke visits, } \\
\mathrm{n}=324\end{array}$} & \multirow[b]{2}{*}{$p$ Value } \\
\hline & $\mathrm{n}$ or Mean & $\%$ or SD & $\mathrm{n}$ or Mean & $\%$ or SD & $\mathrm{n}$ or Mean & $\%$ or SD & \\
\hline \multicolumn{8}{|l|}{ Stroke subtype } \\
\hline Lacunar & 66 & $12 \%$ & 18 & $8 \%$ & 48 & $15 \%$ & $<0.001$ \\
\hline Cardioembolic & 104 & $19 \%$ & 27 & $12 \%$ & 77 & $24 \%$ & \\
\hline Atherosclerotic large-artery stroke & 22 & $4 \%$ & 7 & $3 \%$ & 15 & $5 \%$ & \\
\hline Other/indeterminate etiology & 354 & $65 \%$ & 170 & $77 \%$ & 184 & $57 \%$ & \\
\hline Women & 323 & $59 \%$ & 140 & $63 \%$ & 183 & $56 \%$ & 0.124 \\
\hline Men & 223 & $41 \%$ & 82 & $37 \%$ & 141 & $44 \%$ & \\
\hline \multicolumn{8}{|l|}{ Age, $y$} \\
\hline$<70$ & 18 & $3 \%$ & 0 & $0 \%$ & 18 & $6 \%$ & $<0.001$ \\
\hline $70-75$ & 93 & $17 \%$ & 15 & $7 \%$ & 78 & $24 \%$ & \\
\hline $75-80$ & 180 & $33 \%$ & 71 & $32 \%$ & 109 & $34 \%$ & \\
\hline$>80$ & 255 & $47 \%$ & 136 & $61 \%$ & 119 & $37 \%$ & \\
\hline White & 475 & $87 \%$ & 191 & $86 \%$ & 284 & $88 \%$ & 0.581 \\
\hline Prior CHD (MI or angina) & 229 & $42 \%$ & 99 & $45 \%$ & 130 & $40 \%$ & 0.298 \\
\hline Atrial fibrillation & 55 & $10 \%$ & 17 & $8 \%$ & 38 & $12 \%$ & 0.121 \\
\hline Hypertension, treated or untreated & 388 & $71 \%$ & 161 & $73 \%$ & 227 & $70 \%$ & 0.533 \\
\hline Systolic BP, mm Hg, mean & 143.5 & 24.3 & 145.5 & 24.4 & 142.2 & 24.2 & 0.115 \\
\hline Diastolic BP, mm Hg, mean & 71.6 & 11.6 & 71.9 & 11.4 & 71.3 & 11.7 & 0.547 \\
\hline Diabetes mellitus & 133 & $24 \%$ & 41 & $18 \%$ & 92 & $28 \%$ & 0.008 \\
\hline Hyperlipidemia & 128 & $24 \%$ & 49 & $22 \%$ & 79 & $24 \%$ & 0.609 \\
\hline Total cholesterol, $\mathrm{mmol} / \mathrm{L}$, mean & 5.6 & 1.1 & 5.5 & 1.0 & 5.6 & 1.2 & 0.382 \\
\hline LDL cholesterol, mmol/L, mean & 3.4 & 0.9 & 3.3 & 0.9 & 3.4 & 0.9 & 0.256 \\
\hline HDL cholesterol, mmol/L, mean & 1.4 & 0.4 & 1.4 & 0.4 & 1.3 & 0.4 & 0.086 \\
\hline Body mass index & 26.0 & 4.6 & 25.8 & 4.4 & 26.1 & 4.7 & 0.433 \\
\hline Smoking & 47 & $9 \%$ & 17 & $8 \%$ & 30 & $9 \%$ & 0.512 \\
\hline Income $<\$ 12,000 / y$ & 162 & $31 \%$ & 64 & $30 \%$ & 98 & $32 \%$ & 0.728 \\
\hline
\end{tabular}

$\mathrm{MI}=$ myocardial infarction CHD = coronary heart disease; $\mathrm{BP}=$ blood pressure; LDL = low density lipoprotein; HDL = high density lipoprotein.

the initial year to $5 \%$ in later years (figure 1B). Of recurrent strokes, $73 \%(\mathrm{n}=87)$ were ischemic, $20 \%(\mathrm{n}=25)$ were hemorrhagic, and 7\% $(\mathrm{n}=8)$ were of indeterminate etiology, and the distribution of hemorrhagic, ischemic, and indeterminate strokes among recurrences did not appear to differ over time. In contrast to stroke recurrence, the hazard curves for death and CHD appeared to be relatively constant over time (see figure 1B). During the first year after incident stroke, the incidence per 1,000 personyears was 105.4 (95\% CI $=79.7$ to 139.5$)$ for stroke recurrence and 59.3 (95\% CI $=40.9$ to 85.9) for CHD events. After the first year, the incidence per 1,000 person-years was $52.0(95 \% \mathrm{CI}=41.2$ to 65.6$)$ for stroke and $46.5(95 \%$ $\mathrm{CI}=36.6$ to 59.1) for CHD. CHD rates per 1,000 personyears were 66.1 among subjects with prior CHD and 38.6 among subjects without prior CHD.

Stroke subtype and events. The mortality rate per 1,000 person-years was highest for subjects who had had a cardioembolic stroke $(185.4,95 \%$ CI $=147.7$ to 232.9$)$, intermediate for atherosclerotic stroke $(154.9,95 \% \mathrm{CI}=$ 91.8 to 261.6$)$ and indeterminate stroke $(150.7,95 \% \mathrm{CI}=$
130.6 to 173.8), and lowest for lacunar stroke (119.3, 95\% $\mathrm{CI}=86.5$ to 164.7 ). Compared with lacunar stroke, cardioembolic stroke was associated with a hazard ratio of 1.81 (95\% CI $=1.22$ to 2.70$)$ for death after adjustment for study year, clinic, age, and sex.

For recurrent stroke, the incidence per 1,000 personyears was highest for cardioembolic stroke $(86.6,95 \% \mathrm{CI}=$ 60.6 to 124.9), intermediate for atherosclerotic stroke $(76.4,95 \% \mathrm{CI}=34.3$ to 170.1$)$ and indeterminate stroke $(63.9,95 \% \mathrm{CI}=50.8$ to 80.6$)$, and lowest for lacunar stroke (43.0, 95\% CI $=24.4$ to 75.8). Cardioembolic stroke was associated with significantly increased risk of stroke recurrence (adjusted hazard ratio $=2.01,95 \% \mathrm{CI}=1.03$ to 3.95) compared with lacunar stroke.

The incidence of CHD per 1,000 person-years was 57.9 $(95 \% \mathrm{CI}=37.7$ to 88.7$)$ for cardioembolic stroke, $55.6(95 \%$ $\mathrm{CI}=23.1$ to 137.6$)$ for atherosclerotic stroke, $48.5(95 \%$ $\mathrm{CI}=28.7$ to 81.8$)$ for lacunar stroke, and $47.0(95 \% \mathrm{CI}=$ 36.1 to 61.2 ) for indeterminate stroke. There was no significant association between stroke etiology and incidence of subsequent CHD events. 

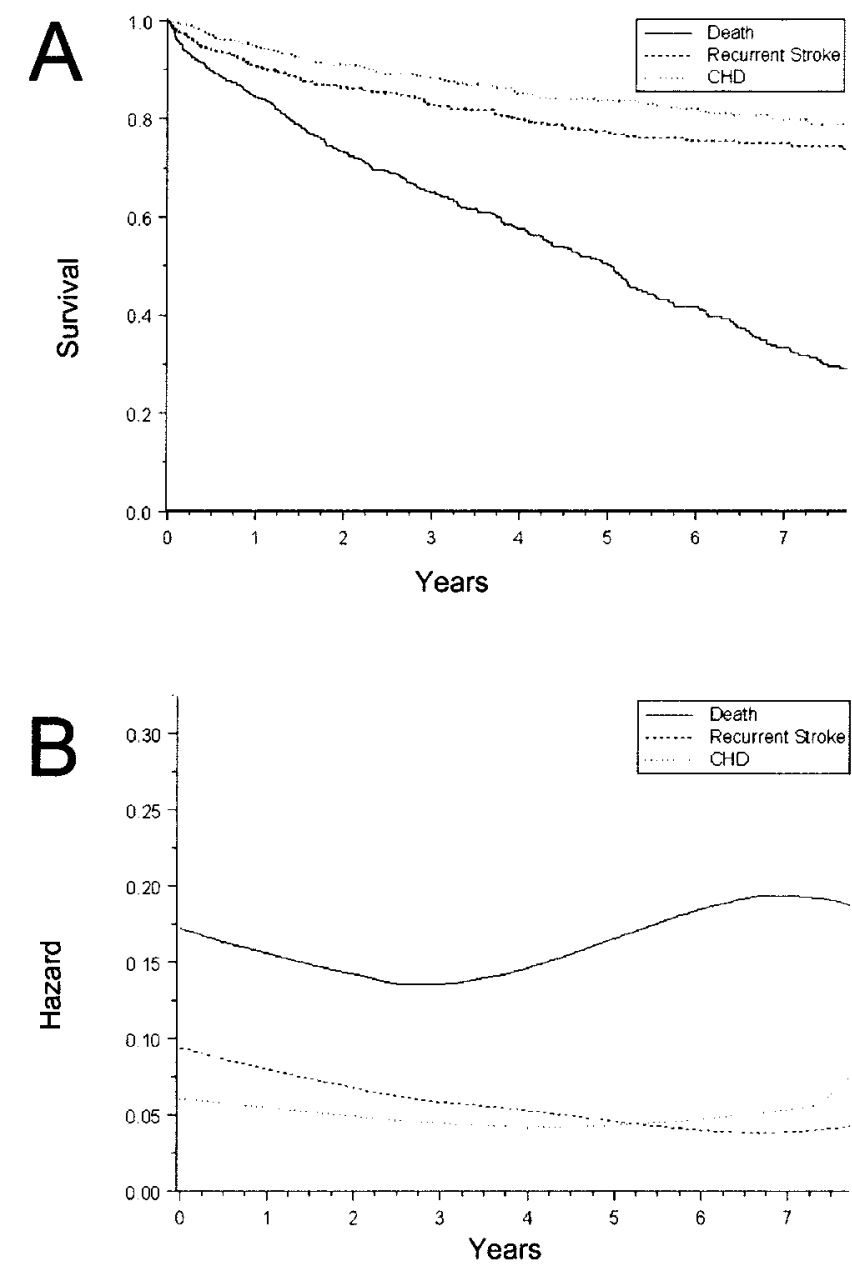

Figure 1. Survival and hazard curves for mortality and vascular events following ischemic stroke. (A) Survival curves for all-cause mortality, recurrent stroke, and coronary heart disease (CHD) events. (B) Smoothed annualized hazard curves for all-cause mortality, recurrent stroke, and CHD events. Time scale is years since initial ischemic stroke. Both survival and hazard curves were censored at 7.75 years, the approximate $90 \%$ percentile for duration of follow-up.
Multivariate analysis of risk factors for events. In multivariate analyses, advanced age was an independent predictor of death and CHD events but not recurrent stroke (table 2). Compared with men, women had a significantly lower risk of death and also tended to have lower risk of CHD events. Other established CVD risk factors, including history of CHD, hypertension, elevated total cholesterol, diabetes, and smoking, had significant or borderlinesignificant associations with death and vascular events in multivariate models. There were no significant differences between white and non-white subjects in risk of death, recurrent stroke, or CHD.

Availability of poststroke visits. For the analysis of medication use after stroke, 104 subjects were ineligible because their ischemic stroke occurred after the last planned CHS visit in 1999. Of the remaining 442 subjects, $324(73 \%)$ completed a poststroke visit. The median time from stroke to the poststroke visit was 292 days. Of poststroke visits, $69 \%$ were conducted at CHS clinics, $19 \%$ were conducted in the participant's home, and $12 \%$ were conducted at a nursing home. Compared with 222 subjects without poststroke visits, the 324 subjects with poststroke visits were less likely to have had indeterminate stroke, were younger, and had higher prevalence of diabetes, but the two groups were otherwise similar (see table 1). Cumulative mortality was similar in subjects with and without poststroke visits (58\% vs 56\%), although median time to death was longer among subjects with visits (4.2 years) compared with those without visits ( 0.8 years). Among patients without poststroke visits, $40 \%$ of the deaths occurred within 6 months and $56 \%$ of the deaths occurred within 1 year of the initial stroke. Cumulative incidence of recurrent stroke (24\% vs $19 \%$ ) and CHD events (21\% vs $13 \%)$ was higher in subjects with poststroke visits.

Use of preventive drug therapies following stroke. After their stroke, fewer than half of the subjects $(47.8 \%)$ were using daily aspirin. Other antiplatelet agents including thienopyridines and dipyridamole were used by $13.5 \%$. While $81.5 \%$ of 243 hypertensive subjects were receiving drug treatment, $40.0 \%$ of treated patients had BP level below 140/90 mm Hg. Use of lipid-lowering therapy among 83 hyperlipidemic patients was $31.3 \%$. Among 98 diabetic

Table 2 Multivariate analyses of risk factors for deaths, recurrent strokes, and CHD events among older adults with ischemic stroke

\begin{tabular}{|c|c|c|c|c|c|c|c|c|c|}
\hline & \multicolumn{3}{|c|}{ Deaths, $\mathrm{n}=313$} & \multicolumn{3}{|c|}{ Recurrent strokes, $\mathrm{n}=120$} & \multicolumn{3}{|c|}{ CHD events, $\mathrm{n}=95$} \\
\hline & $\mathrm{HR}^{*}$ & $95 \% \mathrm{CI}$ & $p$ & $\mathrm{HR}^{*}$ & $95 \% \mathrm{CI}$ & $p$ & $\mathrm{HR}^{*}$ & $95 \% \mathrm{CI}$ & $p$ \\
\hline Age, per $10 \mathrm{y}$ & 2.52 & $2.05,3.10$ & $<0.001$ & 1.00 & $0.70,1.45$ & 0.986 & 1.62 & $1.09,2.41$ & 0.017 \\
\hline Female vs male & 0.78 & $0.61,0.99$ & 0.041 & 0.99 & $0.66,1.48$ & 0.955 & 0.67 & $0.43,1.04$ & 0.074 \\
\hline Nonwhite vs white & 0.94 & $0.63,1.41$ & 0.774 & 0.85 & $0.46,1.57$ & 0.593 & 1.23 & $0.62,2.45$ & 0.554 \\
\hline Atrial fibrillation & 0.82 & $0.55,1.21$ & 0.314 & 1.31 & $0.72,2.38$ & 0.371 & 1.24 & $0.62,2.51$ & 0.544 \\
\hline History of CHD & 1.20 & $0.94,1.52$ & 0.143 & 1.03 & $0.70,1.51$ & 0.878 & 1.73 & $1.12,2.67$ & 0.013 \\
\hline Hypertension & 0.98 & $0.76,1.26$ & 0.890 & 1.51 & $0.96,2.39$ & 0.075 & 1.46 & $0.88,2.42$ & 0.141 \\
\hline Total cholesterol per $\mathrm{mmol} / \mathrm{L}$ & 0.94 & $0.84,1.05$ & 0.268 & 0.94 & $0.78,1.13$ & 0.498 & 1.18 & $1.02,1.36$ & 0.027 \\
\hline Diabetes mellitus & 1.26 & $0.97,1.64$ & 0.082 & 1.59 & $1.07,2.37$ & 0.022 & 1.70 & $1.09,2.64$ & 0.019 \\
\hline Smoking & 1.42 & $0.95,2.11$ & 0.088 & 2.06 & $1.19,3.56$ & 0.010 & 1.42 & $0.69,2.91$ & 0.739 \\
\hline
\end{tabular}

* Adjusted simultaneously for all variables in table, plus stroke subtype, study clinic, and year.

$\mathrm{HR}=$ hazard ratio; CHD = coronary heart disease. 


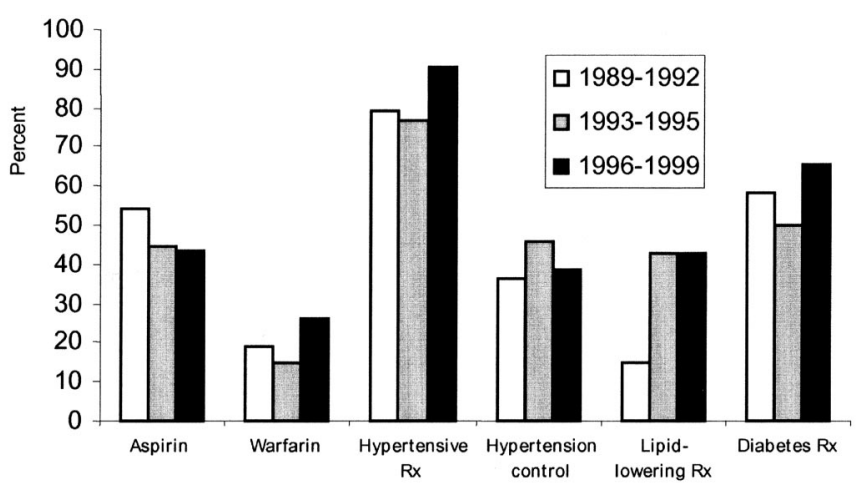

Figure 2. Time trends in use of preventive drug therapies following ischemic stroke, 1989 to 1999.

subjects, $57.0 \%$ were using insulin or oral hypoglycemic agents (see figure E-1 on the Neurology Web site at www. neurology.org).

Time trends in drug utilization following stroke. Over the period 1989 to 1999 , we observed a statistically significant time trend of increasing use of lipid-lowering therapy and warfarin (figure 2). The frequency of lipid-lowering medication use among subjects with hyperlipidemia was $14.7 \%$ in 1989 to $1992,42.9 \%$ in 1993 to 1995 , and $42.9 \%$ in 1996 to 1999. Warfarin was used by $19.2 \%$ in 1989 to $1992,14.7 \%$ in 1993 to 1995 , and $26.1 \%$ in 1996 to 1999 . These trends were significant after adjustment for changes over time in the distribution of strokes by study clinic, age, sex, income, and race.

Clinical factors predicting drug utilization. Multivariate regression models were used to identify independent predictors of preventive therapy use (see tables E-1, E-2, and E-3).

Antiplatelet drugs and warfarin. Aspirin was used by $39.0 \%$ of subjects with cardioembolic stroke, $46.7 \%$ with large-artery stroke, $50.0 \%$ with lacunar stroke, and $51.1 \%$ with indeterminate stroke. Only $42.1 \%$ of subjects older than 75 used aspirin, compared with $61.5 \%$ of subjects 65 to 75 years old. However, because older subjects were more likely to use non-aspirin antiplatelet agents, there was no overall difference in frequency of antiplatelet medication use by age. Aspirin or other antiplatelet medication use was $40.0 \%$ among nonwhite subjects, compared with $57.8 \%$ among white subjects (adjusted OR for nonwhite $=0.54$, $95 \%$ CI $=0.26$ to 1.12 ). Warfarin was used by $52.6 \%$ of patients with atrial fibrillation, $50.7 \%$ of subjects with cardioembolic stroke, $20.0 \%$ with large-artery stroke, $12.5 \%$ with lacunar stroke, and $8.2 \%$ with indeterminate stroke (see table E-1).

Antihypertensive medications. We did not identify any significant predictors of receiving antihypertensive treatment. However, control of BP levels to below 140/90 mm $\mathrm{Hg}$ was achieved in $57.7 \%$ of hypertensive men but only $36.8 \%$ of hypertensive women. Compared with men, women had an adjusted OR of 0.50 (95\% CI $=0.26$ to 0.95 ) for $\mathrm{BP}<140 / 90 \mathrm{~mm} \mathrm{Hg}$ (see table E-2).

Lipid-lowering medications. Older individuals with hyperlipidemia were significantly less likely to use lipidlowering medications. Use was particularly infrequent among those subjects older than 80 , only $8.7 \%$ of whom were using lipid-lowering medications, compared with $40.0 \%$ of younger subjects (compared with $<80$ years, ad- justed OR for $\geq 80$ years $=0.13,95 \% \mathrm{CI}=0.02$ to 0.97 ) (see table E-3).

Diabetes medications. Only $43.3 \%$ of diabetic subjects with income below $\$ 12,000 /$ year were receiving diabetes medications, compared with $62.3 \%$ of those with income $\$ 12,000 /$ year or above. In multivariate analyses, the OR for receiving diabetes treatment was 0.29 (95\% CI $=0.09$ to 0.94 ) among low-income subjects compared to participants with incomes of $\$ 12,000 /$ year or more (see table E-3).

Discussion. We report several findings regarding prognosis and utilization of preventive therapies among older individuals with first ischemic stroke. Over 3.2 years (median) of follow-up, 57.0\% died and $22.0 \%$ had a recurrent stroke. Hemorrhagic strokes accounted for $20 \%$ of recurrent stroke events after ischemic stroke. Compared with previous studies, our results suggest a similar recurrence rate following ischemic stroke, ${ }^{4-7,22}$ but a relatively higher proportion of hemorrhagic strokes among recurrent strokes. $^{6,22}$ Subsequent CHD events occurred in $17.4 \%$, which suggests that CHD was nearly as frequent as recurrent stroke during long-term followup. Advanced age and male sex predicted death and CHD events, although not recurrent stroke. There were no differences in outcomes between white and non-white subjects, possibly due to small numbers of non-white subjects. Approximately 9 months after stroke, antithrombotic agents, lipid-lowering therapy, and diabetes medications were not widely used. There was evidence for less aggressive use of CVD preventive therapies among older subjects, women, and low-income individuals.

Our findings suggest a changing pattern of secondary CVD events over time that has not been identified in prior studies of stroke in the elderly. ${ }^{9}$ Recurrent strokes were approximately twice as frequent as CHD events during the first year after stroke (incidence rate/1,000 person-years $=105.4$ for recurrent stroke and $=59.3$ for $\mathrm{CHD}$ ). Because of declining incidence of stroke over time, the rates of recurrent stroke and $\mathrm{CHD}$ events were similar after the first follow-up year (incidence rate/1,000 personyears $=52.0$ for recurrent stroke and $=46.5$ for CHD). Moreover, our data suggest a higher rate of coronary events after ischemic stroke than previously reported..$^{8-10}$ For example, in a study of 1,518 Medicare enrollees with stroke, the cumulative incidence at 1 year was $6.2 \%$ for recurrent stroke and $1.6 \%$ for acute MI. Between 1 and 3 years, an additional $5.9 \%$ were found to have recurrent stroke and an additional $3.5 \%$ had acute MI. ${ }^{9}$ In our study, the CHD event rate in our study was substantial even among subjects free of prior MI and angina, approaching $4 \%$ per year (38.6/1,000 person-years) in this subgroup. The higher $\mathrm{CHD}$ rate observed in this study may be explained by more complete event identification due to active follow-up of study subjects and review of medical records for all potential CVD events and deaths.

Consistent with previous studies of ischemic 
stroke survivors, we found that rates of mortality and recurrent stroke were lowest among those with lacunar stroke, while those with cardioembolic stroke had relatively high mortality and stroke recurrence rates. ${ }^{4,5,7}$ We did not find major differences in subsequent CHD event rates among patients with lacunar, cardioembolic, atherosclerotic/large-artery strokes, or indeterminate strokes. This suggests a paradox in light of prior findings that asymptomatic cardiac ischemia is more than twice as common among patients with large-artery occlusive stroke compared with those with penetrating artery disease or cryptogenic stroke. ${ }^{23}$ However, our data should be interpreted cautiously because of the large proportion of indeterminate-etiology strokes, the relatively small sample sizes for stroke subtypes (particularly atherosclerotic stroke, $\mathrm{n}=22$ ), and the modest number of CHD events $(n=95)$.

Evidence regarding secondary stroke prevention was evolving during the study period (1989 to 2001), and our data suggested increased use of preventive drug therapies including lipid-lowering drugs and warfarin during this period. Nonetheless, we found that there is substantial room for improvement in the use of medications that may improve prognosis after stroke in the elderly. ${ }^{24}$ Approximately 9 months after stroke, only about half of subjects were using aspirin or other antiplatelet therapies, and 52.6\% of atrial fibrillation patients were treated with warfarin. This may reflect concerns that the benefits of antithrombotic therapy do not outweigh the risks of major bleeding of older patients. ${ }^{25}$ We lacked information on contraindications to antiplatelet and anticoagulant therapy such as peptic ulcer disease, which may be the reason for withholding therapy in some subjects. However, these data are consistent with other reports that these therapies are underutilized, particularly in the elderly.26,27 For example, fewer than $40 \%$ of patients $>65$ years were using aspirin after discharge for stroke in an Ontario cohort from 1993 to $1995 .^{26,27}$ In a study of VA patients with atrial fibrillation from 1998 to 2001, warfarin use among patients $>75$ years was below $50 \%$, even among patients who had the highest stroke risk and were free of contraindications. ${ }^{26,27}$ The Kansas City Stroke Study assessed patients 6 months after stroke (1995 to 1998) and found that antiplatelet agents were used by 55\%, and among those with atrial fibrillation, warfarin was used by $64 \% .^{28}$

Nearly $20 \%$ of hypertensive subjects in this study were not receiving antihypertensive drug therapy after their stroke, consistent with use of antihypertensive treatment in other stroke survivor populations. ${ }^{12,28}$ Only $40 \%$ of drug-treated hypertensive subjects had BP levels controlled to below 140/90 $\mathrm{mm} \mathrm{Hg}$, and women had significantly poorer BP control compared with men. The inadequate treatment of hypertension may reflect controversy regarding the safety of antihypertensive therapy among elderly stroke survivors. The PROGRESS and PATS trials suggested that antihypertensives may reduce subse- quent events in patients with prior stroke or TIA. ${ }^{29,30}$ However, these studies examined relatively young populations (PROGRESS, mean age 64 years and PATS, mean age 60 years), and up to several years had elapsed between their qualifying cerebrovascular event and study enrollment. Despite the lack of specific clinical trial evidence of risk reduction by antihypertensive treatment in older adults with recent stroke, the totality of available evidence favors active treatment and control of BP levels in this group.

Use of lipid-lowering therapy increased over time but was still only approximately $40 \%$ among hypercholesterolemic subjects in the later phase of our study period (1996 to 1999). We found that age was a strong determinant of lipid-lowering therapy use, with medications used by only $8.7 \%$ of hyperlipidemic subjects older than 80. Recent data suggest that interdisciplinary stroke care teams may achieve higher rates of statin use compared to what was observed in this population-based sample. ${ }^{31} \mathrm{We}$ lacked complete information on achieved lipid levels, but previous studies have suggested that fewer than half of stroke survivors meet National Cholesterol Education Program (NCEP) goals for target LDLcholesterol levels. ${ }^{13,32}$ Clinical trials support the use of lipid-lowering therapy in stroke patients up to 80 years old. The PROSPER study, which was a placebo-controlled pravastatin trial conducted specifically in the 70- to 80-year-old population, showed a reduction in vascular events among subjects with prior vascular disease (hazard ratio $=0.78,95 \%$ $\mathrm{CI}=0.66$ to 0.93 ), although there was no clear benefit among those without prior vascular disease (hazard ratio $=0.94,95 \% \mathrm{CI}=0.11$ to 1.15$).{ }^{33} \mathrm{In}$ subgroup analyses from the Heart Protection Study, which included patients 40 to 80 years old, simvastatin reduced the occurrence of coronary events (but not recurrent stroke) among patients with prior cerebrovascular events. ${ }^{34}$ It is necessary to extrapolate from existing data for the 80-years-and-older population because of the lack of clinical trials in this age group.

While tight glycemic control in diabetic patients is likely to reduce CVD events as well as microvascular complications, ${ }^{35}$ only $57 \%$ of diabetic subjects were using insulin or oral hypoglycemic agents after ischemic stroke. Aggressive management of diabetes is costly, logistically difficult to accomplish, and may be associated with risk of hypoglycemia. Our finding that low-income subjects were significantly less likely to be receiving antidiabetes medications may raise concerns about socioeconomic disparities in diabetes care. We did not directly assess hemoglobin A1c or other measures of glycemic control, and it should be noted that use of medications is only one measure of the intensity of diabetes treatment. However, our findings are consistent with prior studies documenting poor glycemic control in diabetic stroke survivors. ${ }^{13}$

Limitations of this study include the inability to 
obtain follow-up visits on all subjects after incident stroke. This may have led us to overestimate the use of preventive medications, because untreated patients may have been more likely to die without completing follow-up visits. Patients without visits were older, possibly due to survival bias, or reflecting the fact that subjects with strokes in the later years of the CHS study had less opportunity to complete poststroke visits before the end of study follow-up. However, information on mortality and CVD events was collected via telephone and by passive means (i.e., database searches), and subjects were also offered the opportunity to conduct study visits in their residence or nursing home, which may have reduced selection biases. Although the study featured standardized collection and expert review of medical record information, we lacked the depth of clinical information on stroke events that has been available in some prior studies. We based our definitions of hypertension, hyperlipidemia, diabetes, and other clinical factors on data collected at standardized study examinations. Community physicians involved in the care of study subjects may have used different criteria for defining CVD risk factors, for example relying upon repeat measurements of $\mathrm{BP}$ and lipids rather than measurements on a single occasion to define hypertension or hyperlipidemia. In addition, we lacked complete information on contraindications, drug intolerance, or other medical factors that may have entered into decisions regarding medication use. Assessment of medications may have been incomplete, and medications that were prescribed, but not used by study subjects, were not captured in our database.

\section{Appendix: Participating CHS Investigators and Institutions}

Steering Committee Chairman: Curt D. Furberg, MD, PhD, Wake Forest University School of Medicine. NHLBI Project Office: Jean Olson, MD, MPH. Wake Forest University School of Medicine: Gregory L. Burke, MD Sharon Jackson, Curt D. Furberg, David S. Lefkowitz, Mary F. Lyles, Cathy Nunn, John Chen, Beverly Tucker, Harriet Weiler. Wake Forest University-ECG Reading Center: Ronald Prineas, MD, PhD. University of California, Davis: John Robbins, MD, MHS. The Johns Hopkins University: Linda P. Fried, MD, MPH. The Johns Hopkins University-MRI Reading Center: David Yousem, MD, MBA. University of Pittsburgh: Lewis H. Kuller, MD, DrPH. University of California, Irvine-Echocardiography Reading Center (baseline): Julius M. Gardin, MD. Georgetown Medical Center-Echocardiography Reading Center (follow-up): John S. Gottdiener, MD. New England Medical Center, Boston-Ultrasound Reading Center: Daniel H. O'Leary, MD. University of Vermont-Central Blood Analysis Laboratory: Russell P. Tracy, PhD. University of Arizona, Tucson-Pulmonary Reading Center Paul Enright, MD. Retinal Reading Center-University of Wisconsin: Ronald Klein, MD. University of Washington-Coordinating Center: Richard A. Kronmal, $\mathrm{PhD}$

\section{References}

1. Muntner P, Garrett E, Klag MJ, Coresh J. Trends in stroke prevalence between 1973 and 1991 in the US population 25 to 74 years of age. Stroke 2002;33:1209-1213.

2. American Heart Association. Heart disease and stroke statistics-2004 update. American Heart Association, 2004.

3. Lovett JK, Coull AJ, Rothwell PM. Early risk of recurrence by subtype of ischemic stroke in population-based incidence studies. Neurology 2004;62:569-573

4. Kolominsky-Rabas PL, Weber M, Gefeller O, Neundoerfer B, Heuschmann PU. Epidemiology of ischemic stroke subtypes according to TOAST criteria: incidence, recurrence, and long-term survival in isch- emic stroke subtypes: a population-based study. Stroke 2001;32:27352740 .

5. Sacco RL, Shi T, Zamanillo MC, Kargman DE. Predictors of mortality and recurrence after hospitalized cerebral infarction in an urban community: the Northern Manhattan Stroke Study. Neurology 1994;44: 626-634.

6. Hillen T, Coshall C, Tilling K, Rudd AG, McGovern R, Wolfe CD. Cause of stroke recurrence is multifactorial: patterns, risk factors, and outcomes of stroke recurrence in the South London Stroke Register. Stroke 2003;34:1457-1463.

7. Petty GW, Brown RD, Jr., Whisnant JP, Sicks JD, O'Fallon WM, Wiebers DO. Ischemic stroke subtypes: a population-based study of functional outcome, survival, and recurrence. Stroke 2000;31:10621068.

8. Dexter DD, Jr., Whisnant JP, Connolly DC, O'Fallon WM. The association of stroke and coronary heart disease: a population study. Mayo Clin Proc 1987;62:1077-1083.

9. Vickrey BG, Rector TS, Wickstrom SL, et al. Occurrence of secondary ischemic events among persons with atherosclerotic vascular disease. Stroke 2002;33:901-906

10. Adams RJ, Chimowitz MI, Alpert JS, et al. Coronary risk evaluation in patients with transient ischemic attack and ischemic stroke: a scientific statement for healthcare professionals from the Stroke Council and the Council on Clinical Cardiology of the American Heart Association/ American Stroke Association. Stroke 2003;34:2310-2322.

11. Glader EL, Stegmayr B, Norrving B, et al. Sex differences in management and outcome after stroke: a Swedish national perspective. Stroke 2003;34:1970-1975.

12. Hillen T, Dundas R, Lawrence E, Stewart JA, Rudd AG, Wolfe CD Antithrombotic and antihypertensive management 3 months after ischemic stroke: a prospective study in an inner city population. Stroke 2000;31:469-475.

13. Mouradian MS, Majumdar SR, Senthilselvan A, Khan K, Shuaib A. How well are hypertension, hyperlipidemia, diabetes, and smoking managed after a stroke or transient ischemic attack? Stroke 2002;33: 1656-1659.

14. Tu JV, Gong Y. Trends in treatment and outcomes for acute stroke patients in Ontario, 1992-1998. Arch Intern Med 2003;163:293-297.

15. Fried LP, Borhani NO, Enright P, et al. The Cardiovascular Health Study: design and rationale. Ann Epidemiol 1991;1:263-276.

16. Furberg CD, Manolio TA, Psaty BM, et al. Major electrocardiographic abnormalities in persons aged 65 years and older (the Cardiovascular Health Study). Cardiovascular Health Study Collaborative Research Group. Am J Cardiol 1992;69:1329-1335.

17. Psaty BM, Lee M, Savage PJ, Rutan GH, German PS, Lyles M. Assess ing the use of medications in the elderly: methods and initial experience in the Cardiovascular Health Study. The Cardiovascular Health Study Collaborative Research Group. J Clin Epidemiol 1992;45:683-692.

18. Cushman M, Cornell ES, Howard PR, Bovill EG, Tracy RP. Laboratory methods and quality assurance in the Cardiovascular Health Study. Clin Chem 1995;41:264-270.

19. Ives DG, Fitzpatrick AL, Bild DE, et al. Surveillance and ascertainment of cardiovascular events. The Cardiovascular Health Study. Ann Epidemiol 1995;5:278-285.

20. Prevention of stroke by antihypertensive drug treatment in older persons with isolated systolic hypertension. Final results of the Systolic Hypertension in the Elderly Program (SHEP). SHEP Cooperative Research Group. JAMA 1991;265:3255-3264.

21. Longstreth WT, Jr., Bernick C, Fitzpatrick A, et al. Frequency and predictors of stroke death in 5,888 participants in the Cardiovascular Health Study. Neurology 2001;56:368-375.

22. Meissner I, Whisnant JP, Garraway WM. Hypertension management and stroke recurrence in a community (Rochester, Minnesota, 19501979). Stroke 1988;19:459-463.

23. Chimowitz MI, Poole RM, Starling MR, Schwaiger M, Gross MD. Frequency and severity of asymptomatic coronary disease in patients with different causes of stroke. Stroke 1997;28:941-945.

24. Hankey GJ, Warlow CP. Treatment and secondary prevention of stroke: evidence, costs, and effects on individuals and populations. Lancet 1999;354:1457-1463

25. Albers GW, Dalen JE, Laupacis A, Manning WJ, Petersen P, Singer DE. Antithrombotic therapy in atrial fibrillation. Chest 2001;119:194S$206 \mathrm{~S}$.

26. Brophy MT, Snyder KE, Gaehde S, Ives C, Gagnon D, Fiore LD. Anticoagulant use for atrial fibrillation in the elderly. J Am Geriatr Soc 2004;52:1151-1156.

27. Holroyd-Leduc JM, Kapral MK, Austin PC, Tu JV. Sex differences and similarities in the management and outcome of stroke patients. Stroke 2000;31:1833-1837.

28. Rigler SK, Webb MJ, Patel AT, Lai SM, Duncan PW. Use of antihypertensive and antithrombotic medications after stroke in communitybased care. Ann Pharmacother 2001;35:811-816.

29. Post-stroke antihypertensive treatment study. A preliminary result. PATS Collaborating Group. Chin Med J (Engl) 1995;108:710-717. 
30. Randomised trial of a perindopril-based blood-pressure-lowering regimen among 6,105 individuals with previous stroke or transient ischaemic attack. Lancet 2001;358:1033-1041.

31. Carswell JL, Beard KA, Chevrette MM, Pardue CN, Hess DC, Fagan SC. Tracking trends in secondary stroke prevention strategies. Ann Pharmacother 2004;38:215-219.

32. Third Report of the National Cholesterol Education Program (NCEP Expert Panel on Detection, Evaluation, and Treatment of High Blood Cholesterol in Adults (Adult Treatment Panel III) final report. Circulation $2002 ; 106: 3143-3421$.
33. Shepherd J, Blauw GJ, Murphy MB, et al. Pravastatin in elderly individuals at risk of vascular disease (PROSPER): a randomised controlled trial. Lancet 2002;360:1623-1630.

34. Collins R, Armitage J, Parish S, Sleight P, Peto R. Effects of cholesterol-lowering with simvastatin on stroke and other major vascular events in 20536 people with cerebrovascular disease or other highrisk conditions. Lancet 2004;363:757-767.

35. Tight blood pressure control and risk of macrovascular and microvascular complications in type 2 diabetes: UKPDS 38. UK Prospective Diabetes Study Group. BMJ 1998;317:703-713.

Neurolmages

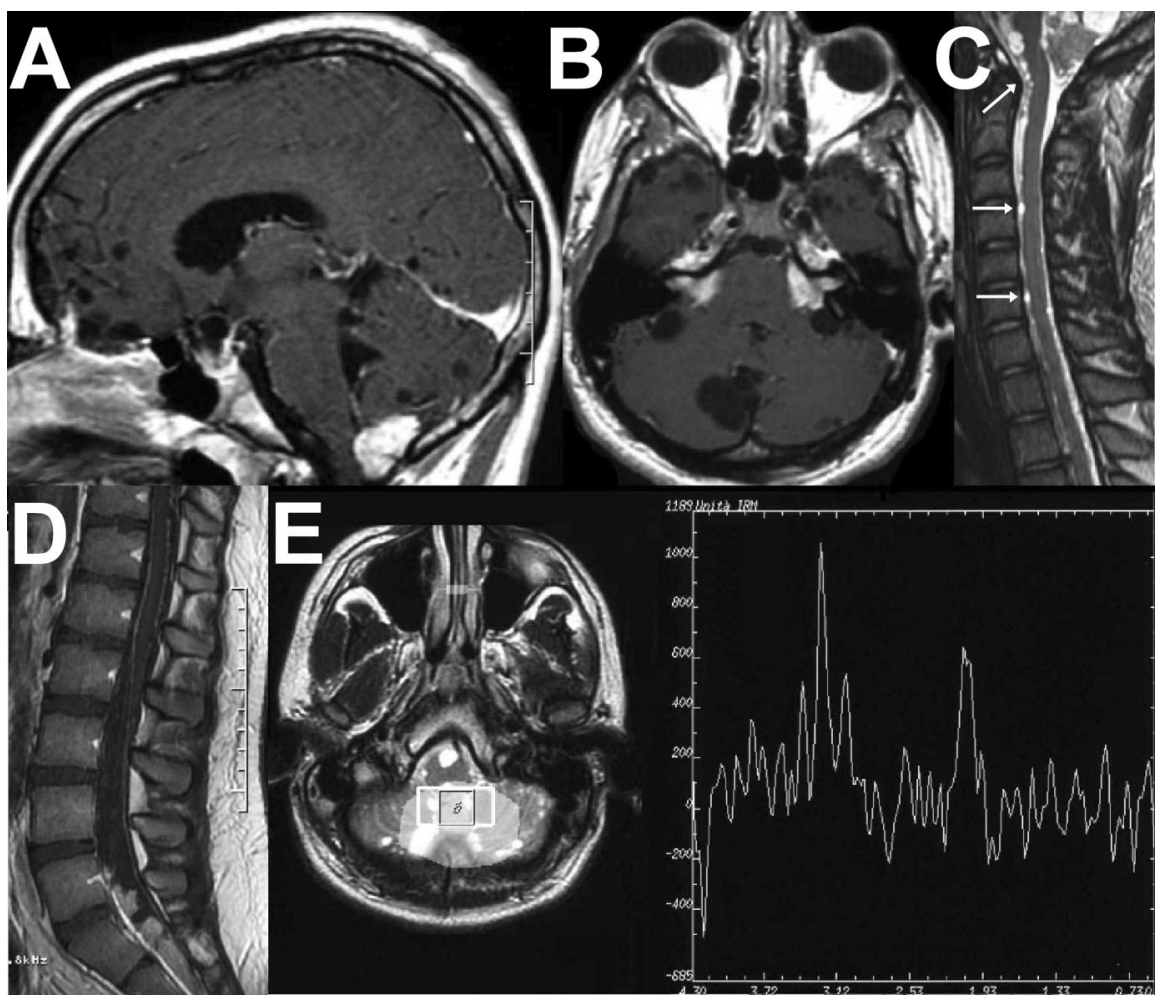

Figure. Cranial sagittal (A) and axial (B), lumbosacral sagittal (D) T1weighted postcontrast and cervicodor sal T2-weighted $(C)$ images showing solid lesions in the cisterna magna, both cerebellopontine angles and the lumbosacral space, as well as multiple craniospinal cystic appearing nonenhancing lesions ( $A, B$, arrows in $C$ ). $M R$ spectroscopy (E): in the solid cisterna magna lesion the relative metabolic peak ratio of Cho/NAA is inverted (1.39, normal values $<1)$. No lactate peak was evident.

\section{Diffuse craniospinal choroid plexus papilloma with involvement of both cerebellopontine angles}

F. Doglietto, MD; L. Lauretti, MD; T. Tartaglione, $M D$; M. Gessi, MD; E. Fernandez, MD; and G. Maira, MD, Rome, Italy

In a 16-year-old boy with headache; deficit of third, sixth, and eight left cranial nerves; and retinal hemorrhagic papilledema, MRI showed multiple craniospinal lesions (figure 1, A through D) Cysticercosis was suspected, but MRI spectroscopy suggested a neoplasm (figure $1 \mathrm{E}$ ). The surgically removed cisterna magna solid and cystic lesions were a choroid plexus papilloma ${ }^{1,2}$ (figure $2, \mathrm{~A}$ and $\mathrm{B})$.

The boy remains well after 1 year; only left hypoacusia persists. Serial MRIs and audiometry have shown no evidence of disease progression.

Disclosure: The authors report no conflicts of interest.

Address correspondence and reprint requests to Dr. Francesco Doglietto, Institute of Neurosurgery, Catholic University School of Medicine, L.go A. Gemelli, 8, 00168 Rome, Italy; e-mail: frandog@tiscali.it

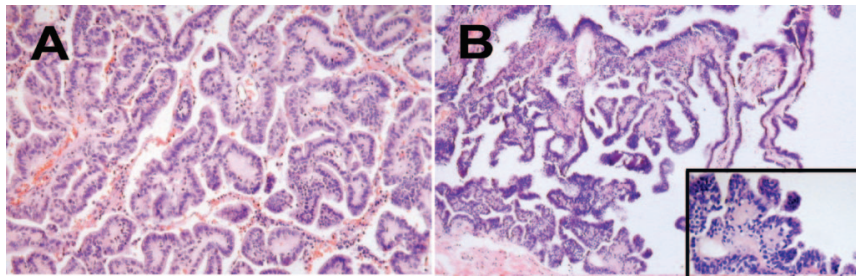

Figure 2. Histopathologic features of the cisterna magna parenchymal (A) and cystic (B) lesions, both of a benign, typical choroid plexus papilloma, composed of papillae lined by columnar epithelium (insert) (A: 250×; B: 200×; insert: $350 \times$; hematoxylin-eosin staining). Ki67 index was less than $1 \%$.

Copyright $\odot 2005$ by AAN Enterprises, Inc.

1. Aguzzi A, Brander S, Paulus W. Choroid plexus tumours. In: Kleihues P Cavenee WK, eds. Tumours of the nervous system: Pathology and genetics. Lyon, France: IARC Press, 2000: 83-86.

2. Leblanc R, Bekhor S, Melanson D, Carpenter S. Diffuse craniospinal seeding from a benign fourth ventricle choroid plexus papilloma: case report. J Neurosurg 1998;88:757-760. 


\section{Neurology}

\section{Diffuse craniospinal choroid plexus papilloma with involvement of both cerebellopontine angles}

F. Doglietto, L. Lauretti, T. Tartaglione, et al. Neurology 2005;65;842

DOI 10.1212/01.wnl.0000167136.64675.9e

This information is current as of September 26, 2005

\section{Updated Information \&} Services

References

Permissions \& Licensing

Reprints including high resolution figures, can be found at: http://n.neurology.org/content/65/6/842.full

This article cites 1 articles, 0 of which you can access for free at: http://n.neurology.org/content/65/6/842.full\#ref-list-1

Information about reproducing this article in parts (figures,tables) or in its entirety can be found online at:

http://www.neurology.org/about/about_the_journal\#permissions

Information about ordering reprints can be found online: http://n.neurology.org/subscribers/advertise

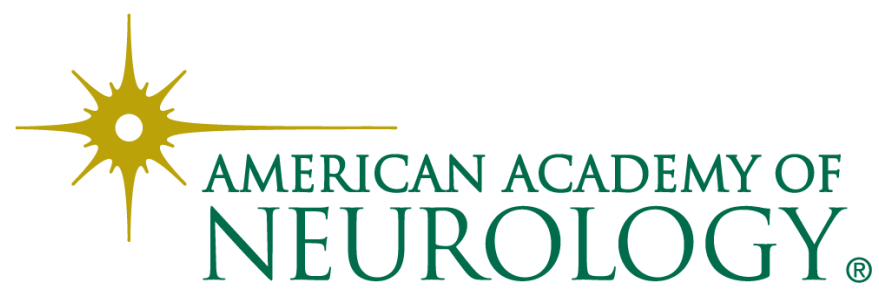

